\title{
Joint Angle and Frequency Estimation Using Multiple-Delay Output Based on ESPRIT
}

\author{
Wang Xudong \\ Department of Electronic Engineering, Nanjing University of Aeronautics and Astronautics, Nanjing 210016, China \\ Correspondence should be addressed to Wang Xudong, xudong@nuaa.edu.cn
}

Received 29 August 2010; Revised 6 December 2010; Accepted 21 December 2010

Academic Editor: Ricardo Merched

Copyright (c) 2010 Wang Xudong. This is an open access article distributed under the Creative Commons Attribution License, which permits unrestricted use, distribution, and reproduction in any medium, provided the original work is properly cited.

This paper presents a novel ESPRIT algorithm-based joint angle and frequency estimation using multiple-delay output (MDJAFE). The algorithm can estimate the joint angles and frequencies, since the use of multiple output makes the estimation accuracy greatly improved when compared with a conventional algorithm. The useful behavior of the proposed algorithm is verified by simulations.

\section{Introduction}

Antenna array has been used in many fields such as radar, sonar, electron reconnaissance and seismic data processing. The direction-of-arrival- (DOA-) estimation of signals impinging on an array of sensors is a fundamental problem in array processing [1-5]. Angle estimation and frequency estimation $[6,7]$ are two key problems in the signal processing field. The problem of joint DOA and frequency estimation arises in the applications of radar, wireless communications and electron reconnaissance. For example, these parameters can be applied to locate the radars and to locate pilot tones in electron reconnaissance systems [8]. Furthermore, a precise estimation of these parameters is helpful to attain a better pulse descriptor word (PDW) and thus enhances the system performance. Optimal techniques based on maximum likelihood [9] are often applicable but might be computationally prohibitive. Some ESPRIT-based joint angle and frequency estimation methods have been proposed in [10-14]. Zoltowski and Mathew [10] discuss this problem in the context of radar applications. ProESPRIT is proposed to estimate angle and frequency. Haardt and Nossek [11] discuss the problem in the context of mobile communications for space division multiple access applications. Their method is based on Unitary-ESPRIT, which involves a certain transformation of the data to real valued matrices. Multi resolution ESPRIT is used for joint angle frequency estimation in [12]. ESPRIT method is used for frequency and angle estimation under uniform circular array in $[13,14]$. References $[15,16]$ proposed the trilinear decomposition method for joint angle and frequency estimation method. The other joint angle and frequency estimation method is proposed in [17-24].

This paper uses multiple-delay output, so as to achieve the purpose of improving estimation accuracy. This algorithm has the improved performance compared with conventional method. The proposed algorithm is applicable to uniform linear array.

Note 1 . We denote by $(\cdot)^{T}$ the matrix transpose, and by $(\cdot)^{H}$ the matrix conjugate transpose. The notation $(\cdot)^{+}$refers to the Moore-Penrose inverse (pseudoinverse).

\section{The Data Model}

There are $K$ sources to reach uniform linear array with $M$ elements. Suppose that the $i$ th source has a carrier frequency $f_{i}$. The signal received at the $m$ th antenna is

$$
x_{m}(t)=\sum_{i=1}^{K} \mathrm{e}^{j 2 \pi(m-1) d f_{i} \sin \left(\theta_{i}\right) / c} s_{i}(t)
$$

where $\theta_{i}$ is direction of arrival (DOA) of the $i$ th signal, and $d$ is array spacing. $s_{i}(t)$ is the narrow-band signal of the $i$ th source. In order to estimate frequency, we add $P-1$ delayed 


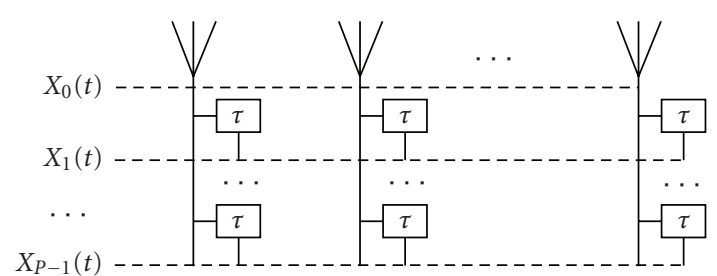

Figure 1: The received signal with delayed output.

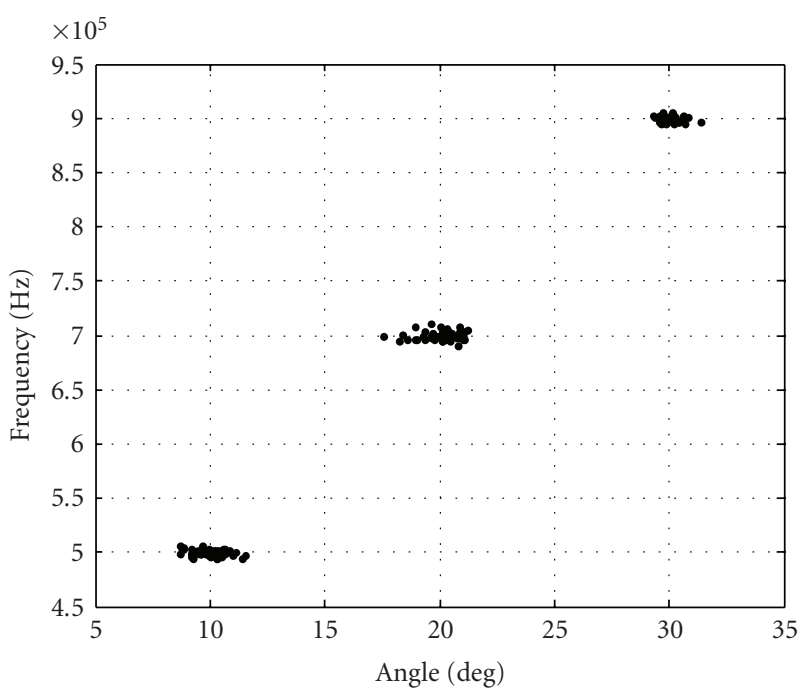

Figure 2: Angle-frequency scatter, SNR $=15 \mathrm{~dB}$.

outputs for the received signal of array antenna, as shown in Figure 1. We suppose that $0<(P-1) \tau<1 / \max \left(f_{i}\right)$.

The delayed signal for (1) with delay $\tau$ is

$$
\begin{aligned}
x_{m}(t-\tau) & =\sum_{i=1}^{K} \mathrm{e}^{j 2 \pi(m-1) d f_{i} \sin \left(\theta_{i}\right) / c} s_{i}(t-\tau) \\
& =\sum_{i=1}^{K} \mathrm{e}^{j 2 \pi(m-1) d f_{i} \sin \left(\theta_{i}\right) / c} s_{i}(t) \mathrm{e}^{-j 2 \pi f_{i} \tau},
\end{aligned}
$$

where $c$ is velocity of light. We assume that channel state information is constant for $N$ symbols. The received signal of array antennas without delay can be denoted as

$$
X_{0}=A S
$$

where the source matrix $\mathbf{S}$ and the direction matrix $\mathbf{A}$ are shown as follows

$$
\begin{gathered}
\mathbf{S}=\left[\begin{array}{cccc}
\mathbf{s}_{1} & \mathbf{s}_{2} & \cdots & \mathbf{s}_{K}
\end{array}\right]^{T} \in \mathbb{C}^{K \times N}, \\
A=\left[\begin{array}{cccc}
1 & 1 & \cdots & 1 \\
\mathrm{e}^{-j \alpha_{1}} & \mathrm{e}^{-j \alpha_{2}} & \cdots & \mathrm{e}^{-j \alpha_{K}} \\
\vdots & \vdots & \ddots & \vdots \\
\mathrm{e}^{-j(M-1) \alpha_{1}} & \mathrm{e}^{-j(M-1) \alpha_{2}} & \cdots & \mathrm{e}^{-j(M-1) \alpha_{K}}
\end{array}\right],
\end{gathered}
$$

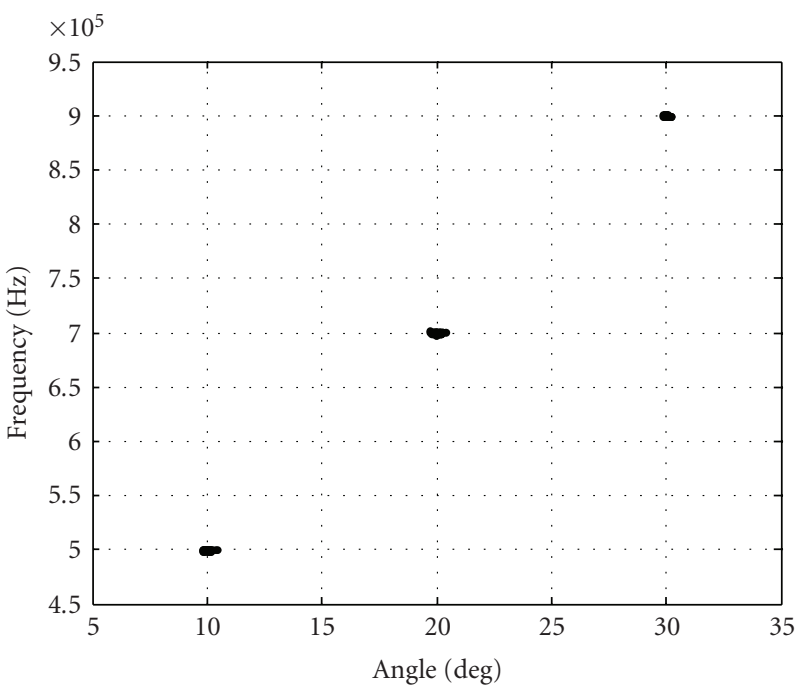

FIgURE 3: Angle-frequency scatter, SNR $=30 \mathrm{~dB}$.

where $\alpha_{k}=2 \pi d f_{k} \sin \left(\theta_{k}\right) / c, k=1,2, \ldots, K$. According to (5), we define

$$
A=\left[\begin{array}{l}
A_{1} \\
a_{M}
\end{array}\right]=\left[\begin{array}{l}
a_{1} \\
A_{2}
\end{array}\right],
$$

where $A_{1}$ is the first $M-1$ rows of $A, a_{M}$ is the last row of $A$. $A_{2}$ is the second to the $M$ th row of $A, a_{1}$ is the first row of $A$. The delayed signal for (2) with $\tau$ can be denoted as

$$
X_{1}=A \Phi S,
$$

where

$$
\Phi=\operatorname{diag}\left\{e^{-j \beta_{1}}, e^{-j \beta_{2}}, \ldots, e^{-j \beta_{K}}\right\},
$$

where $\beta_{k}=2 \pi f_{k} \tau, k=1,2, \ldots, K$.

The delayed signal for (2) with $p \tau$ can be denoted as

$$
X_{p}=A \Phi^{p} S, \quad p=0,1, \ldots, P-1 .
$$

According to (3), (7), and (9), we define

$$
X=\left[\begin{array}{c}
X_{0} \\
X_{1} \\
X_{P-1}
\end{array}\right]=\left[\begin{array}{c}
A \\
A \Phi \\
A \Phi^{P-1}
\end{array}\right] S .
$$

\section{Joint Angle and Frequency Estimation}

We can use received signal to attain the direction matrix $\mathbf{A}$ and the delay matrix $\boldsymbol{\Phi}$, and then estimate angle and frequency. The covariance matrix of the received signal can be reconstructed via $R_{x}=X X^{H}$. Using eigenvalue decomposition of $R_{x}$, we can get the signal subspace $E_{s}$. In the free-noise case, $E_{s}$ can be denoted as

$$
E_{s}=\left[\begin{array}{c}
A \\
A \Phi \\
A \Phi^{P-1}
\end{array}\right] T,
$$




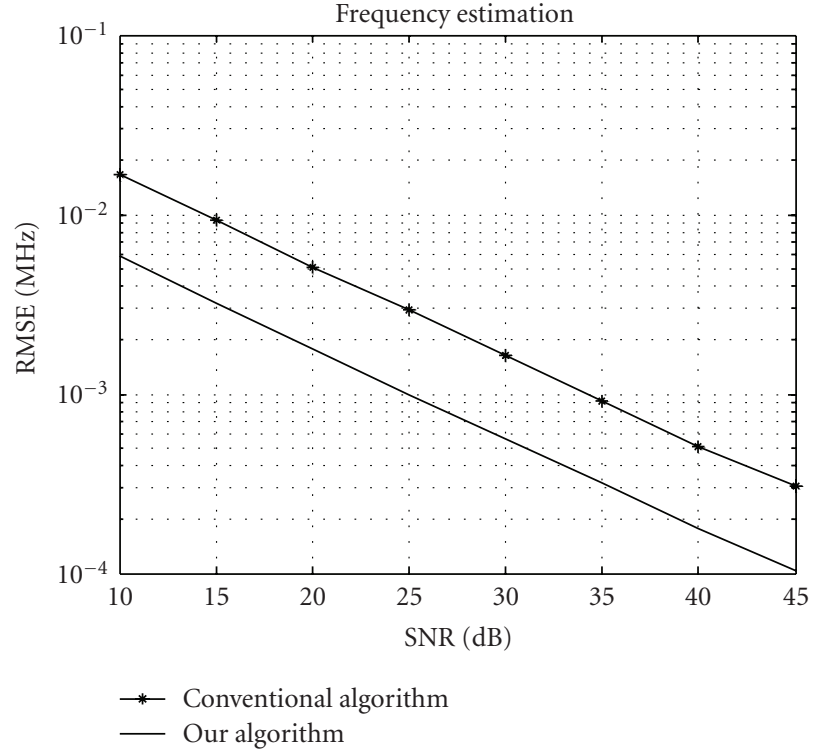

(a)

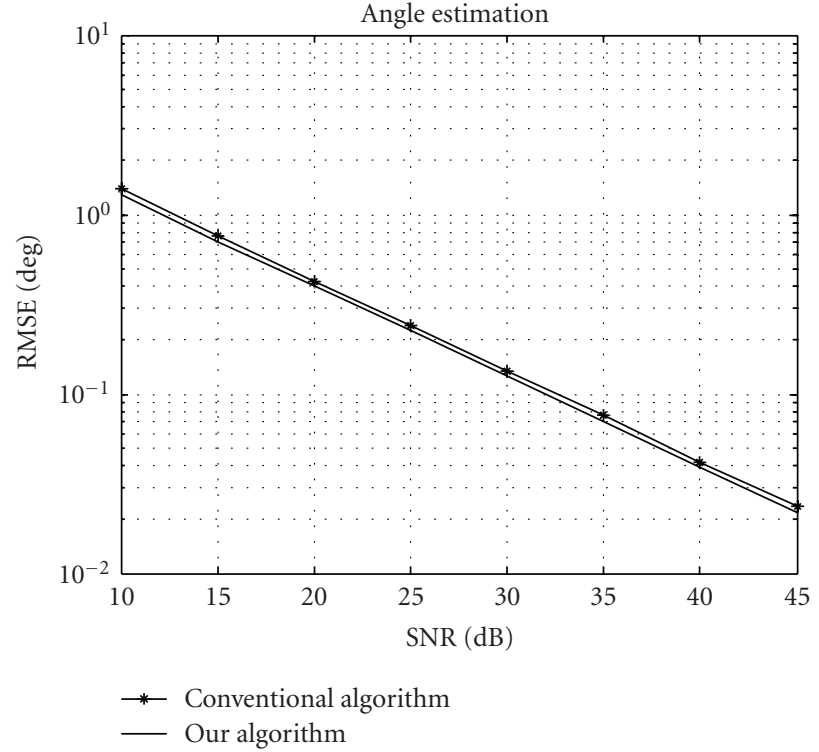

(b)

FIGURE 4: Angle-frequency estimation performance comparison.

3.1. Frequency Estimation. According to (11), we define $E_{1}$ and $E_{2}$

$$
E_{1}=\left[\begin{array}{c}
A \\
A \Phi \\
A \Phi^{P-2}
\end{array}\right] T, \quad E_{2}=\left[\begin{array}{c}
A \Phi \\
A \Phi^{2} \\
A \Phi^{P-1}
\end{array}\right] T
$$

According to (12),

$$
E_{2}=\left[\begin{array}{c}
A \Phi \\
A \Phi^{2} \\
A \Phi^{P-1}
\end{array}\right] T=\left[\begin{array}{c}
A \\
A \Phi \\
A \Phi^{P-2}
\end{array}\right] T T^{-1} \Phi T=E_{1} T^{-1} \Phi T
$$

Let $\Psi=T^{-1} \Phi T$, so $\Psi=E_{1}{ }^{+} E_{2}$. Because $\Psi$ has the same eigenvalues as $\boldsymbol{\Phi}$, we use eigenvalue decomposition on $\Psi$ to get $\beta_{k}, k=1,2, \ldots, K$ and then estimate frequency $f_{k}, k=$ $1,2, \ldots, K$. Using eigenvalue decomposition of $\Psi$, we can get the eigenvalues $\lambda_{k}, k=1,2, \ldots, K$.

$$
\hat{f}_{k}=\frac{1}{2 \pi \tau} \operatorname{angle}\left(\lambda_{k}\right)
$$

where angle $(\cdot)$ denotes taking the phase angles.

3.2. Angle Estimation. According to (11), take first to ( $M-$ $1)$ th row of $E_{s}$ to get $E_{3}$, which is shown as follows

$$
E_{3}=A_{1} T \in \mathbb{C}^{(M-1) \times K} .
$$

Take second to $M$ th row of $E_{s}$ to get $E_{4}$,

$$
E_{4}=A_{2} T \in \mathbb{C}^{(M-1) \times K} .
$$

We can get

$$
E_{4}=E_{3} T^{-1} \phi T,
$$

where

$$
\varphi=\operatorname{diag}\left\{e^{-j \alpha_{1}}, e^{-j \alpha_{2}}, \ldots, e^{-j \alpha_{K}}\right\} \in \mathbb{C}^{K \times K},
$$

where $\alpha_{k}=2 \pi d f_{k} \sin \theta_{k} / c, k=1,2, \ldots, K$.

Let $\Omega=T^{-1} \varphi T$, so $\Omega=E_{3}{ }^{+} E_{4}$. Because $\Omega$ has the same eigenvalues as $\varphi$, we use eigenvalue decomposition on $\Omega$ to get $\alpha_{k}, k=1,2, \ldots, K$. And then estimate $\theta_{k}, k=$ $1,2, \ldots, K$. Using eigenvalue decomposition of $\Omega$, we can get the eigenvalues $\xi_{k}, k=1,2, \ldots, K$.

$$
\hat{\theta}_{k}=\arcsin \left(\frac{c}{2 \pi f_{k} d} \operatorname{angle}\left(\xi_{k}\right)\right) .
$$

In contrast to ESPRIT algorithm [13], this algorithm has a high computational load, which is usually dominated by formation of the covariance matrix, matrix inversion and calculation of EVD. The major computational complexity of this algorithm is $O\left(M^{2} P^{2} N+M^{3} P^{3}+K^{3}\right)$, while ESPRIT requires $O\left(M^{2} N+M^{3}+K^{3}\right)$, where $M, P, N$, and $K$ are the number of antennas, delays, snapshots, and sources.

\section{Simulation Results}

We present Monte Carlo simulations that are used to assess the angle and frequency estimation performance of MDJAFE algorithm. The number of Monte Carlo trials is 1000 . Note that $M$ is the number of antennas; $P$ is the number of the delays; $N$ is the number of snapshots; $K$ is the number of the sources.

Define RMSE $=\sqrt{(1 / 1000) \sum_{m=1}^{1000}\left[a_{m}-a_{0}\right]^{2}}$, where $a_{m}$ is the estimated angle/frequency, and $a_{0}$ is the perfect angle/ frequency. 


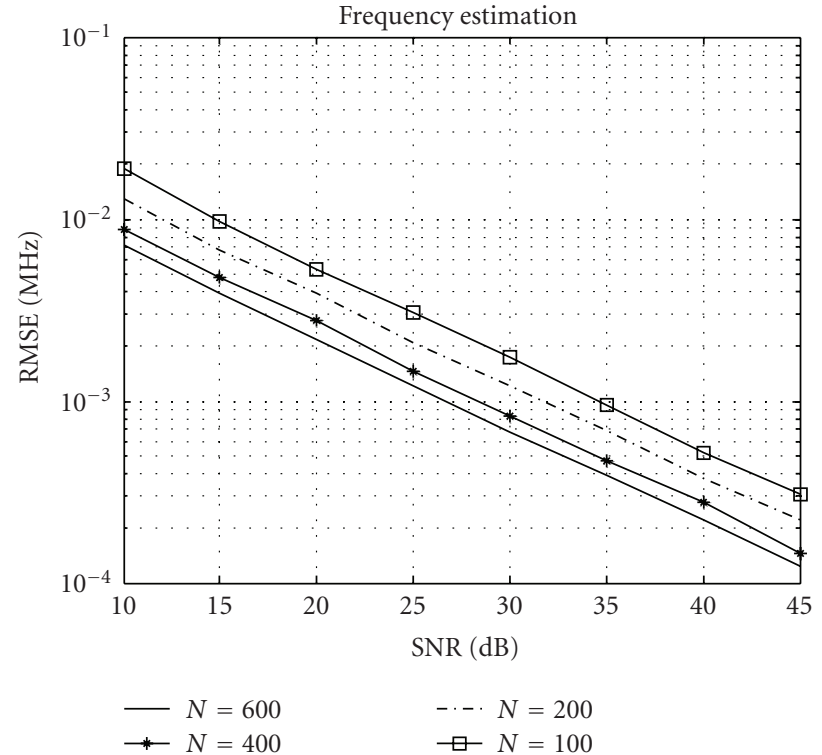

(a)

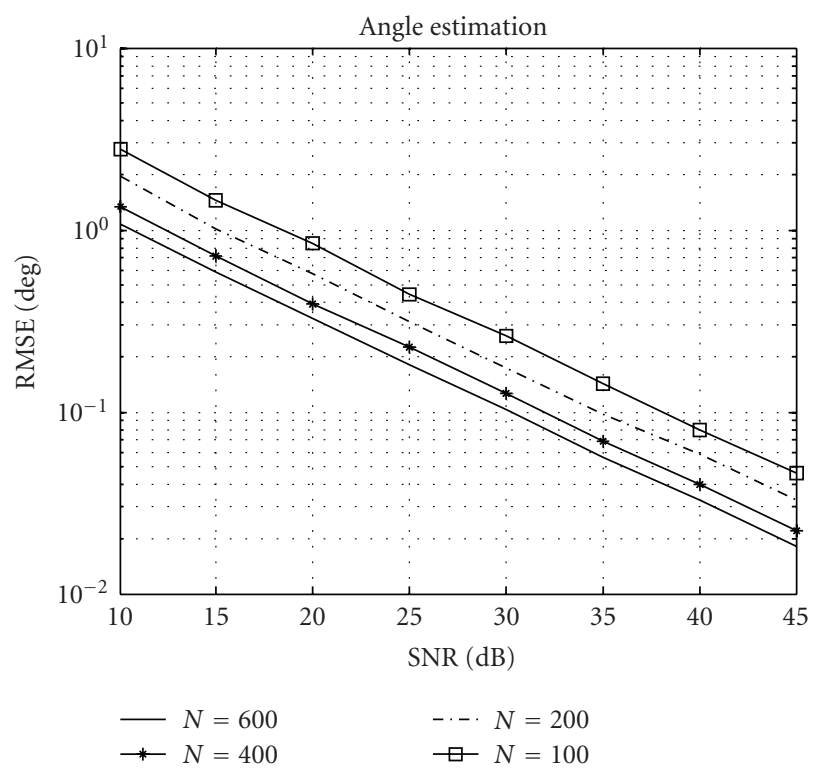

(b)

Figure 5: Angle-frequency estimation with different snapshot $N$.

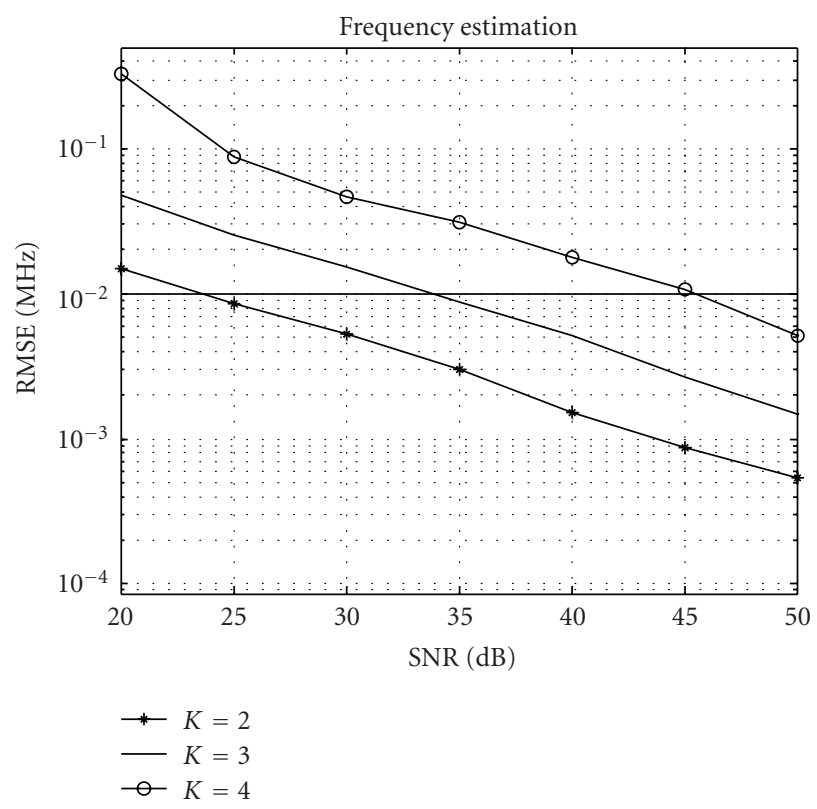

(a)

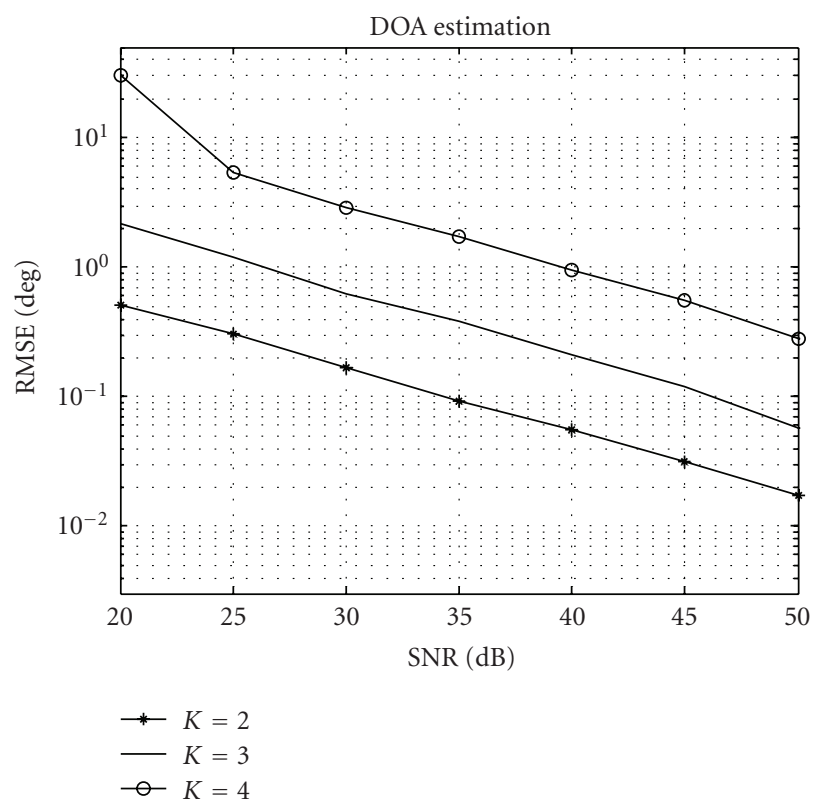

(b)

Figure 6: Angle-frequency estimation with different sources.

Simulation 1. The performance of this proposed algorithm is investigated. $M=12, K=3, P=3$, and $N=400$ in this simulation. Their DOAs are $10^{\circ}, 20^{\circ}$ and $30^{\circ}$ ), and their carrier frequencies are $500 \mathrm{kHZ}, 700 \mathrm{kHZ}$ and $900 \mathrm{kHZ}$. Figure 2 shows the performance of this proposed algorithm with $\mathrm{SNR}=15 \mathrm{~dB}, 30 \mathrm{~dB}$. From Figures 2 and 3 we find that this proposed algorithm works well.

Simulation 2. We compare this proposed algorithm with conventional method [13] which is without delay. $M=12$,
$K=3, P=3$, and $N=400$ in this simulation. From Figure 4 we find that this proposed algorithm has better angle-frequency estimation performance than conventional method.

Simulation 3. MDJAFE algorithm performance under different snapshots $N$ is investigated in this simulation. $M=12$, $K=3$, and $P=3$ in this simulation. Figure 5 shows the angle-frequency estimation performance under different $N$. 


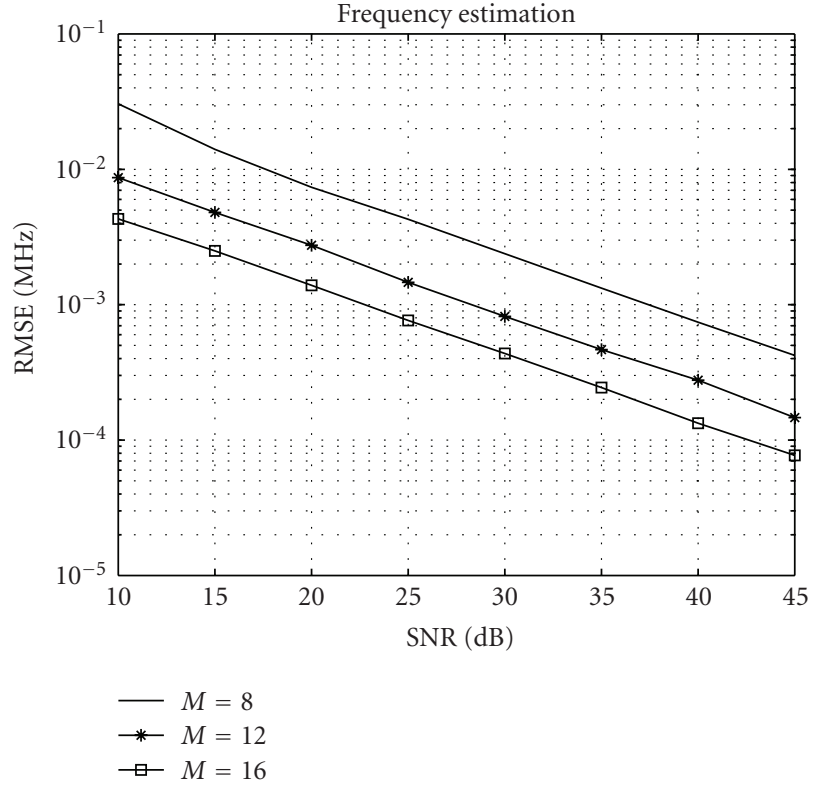

(a)

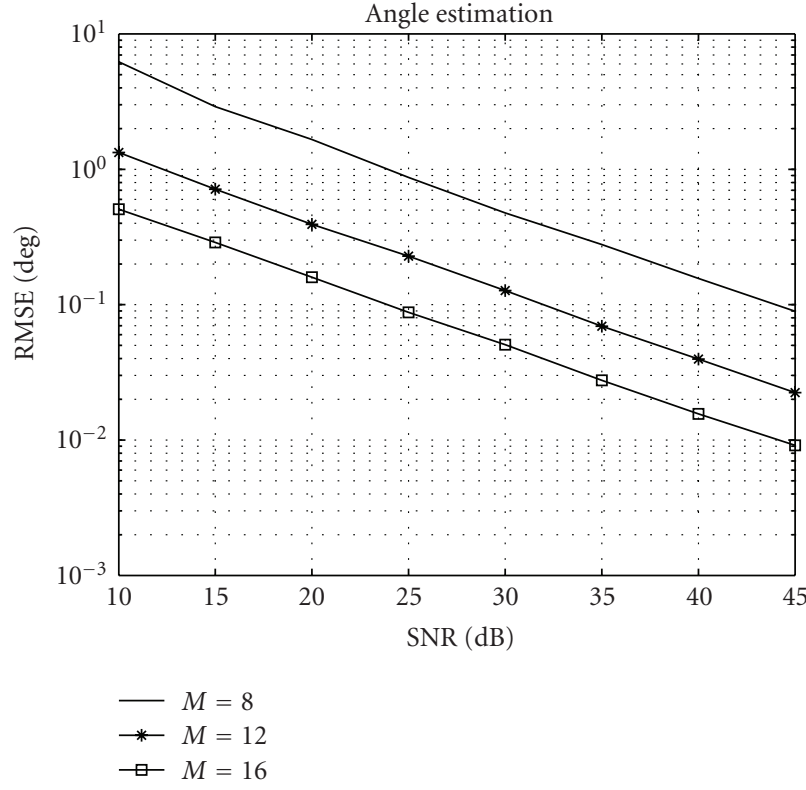

(b)

Figure 7: Angle-frequency estimation with different antennas.

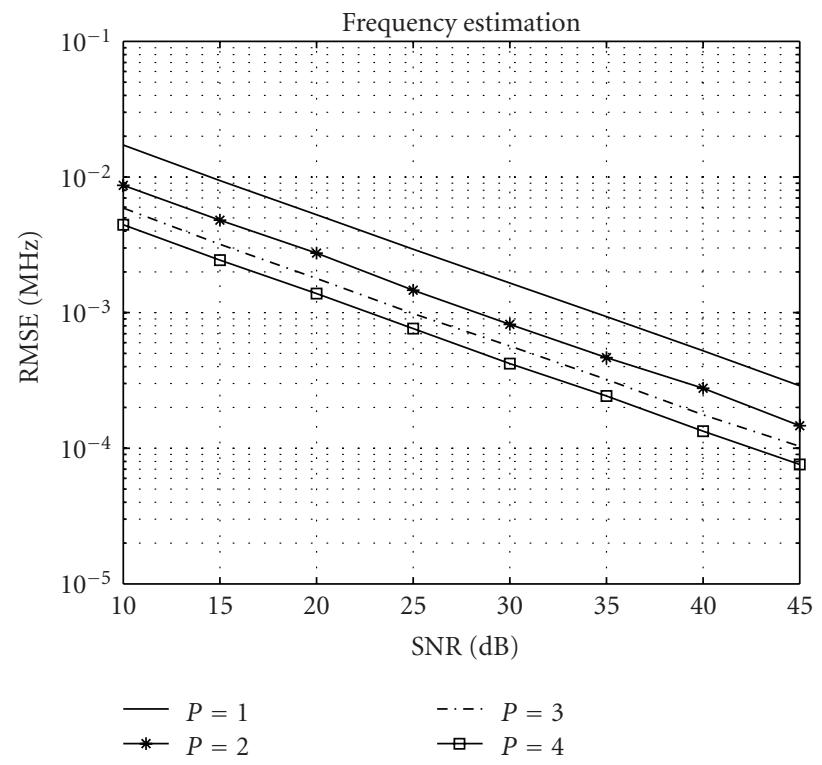

(a)

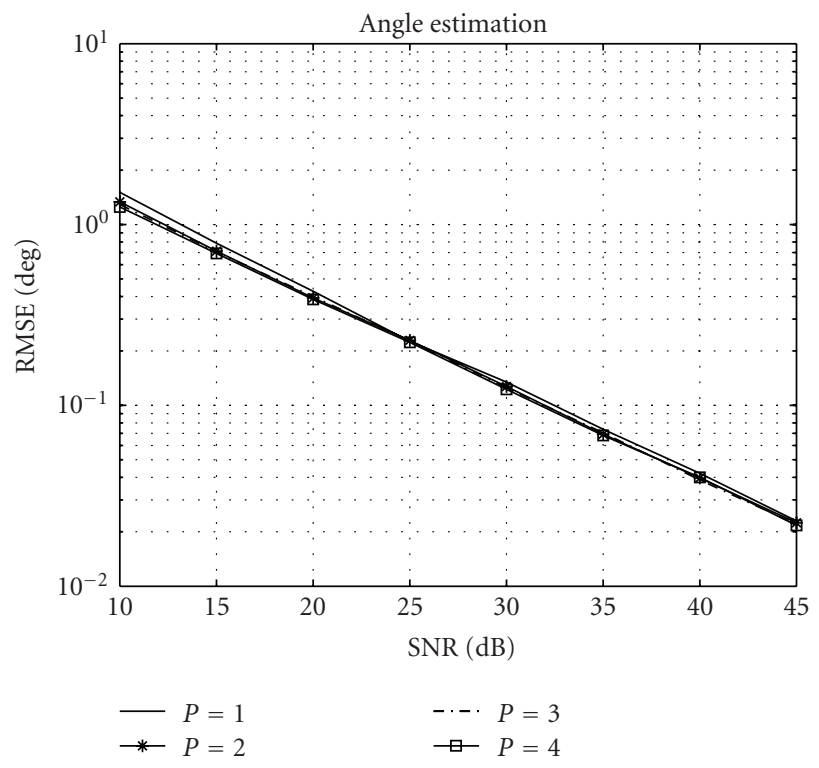

(b)

FIGURE 8: Angle-frequency estimation with different delay number.

We find that the angle-frequency estimation performance of MDJAFE algorithm is improved with $N$ increasing.

Simulation 4. The performance of this algorithm under different source number $K$ is investigated in the simulation. $M=12, P=3$, and $N=400$ in this simulation. The source number $K$ is set to 2, 3, and 4. MDJAFE algorithm has different performance under different source numbers, as shown in Figure 6. From Figure 6, we find that angle and frequency estimation performance of MDJAFE algorithm degrades with the increase of the source number $K$.

Simulation 5. The performance of this algorithm under different antenna number $M$ is investigated in the simulation. $K=3, P=3$, and $N=400$ in this simulation. The antenna number $M$ is set to 8,12 , and 16. MDJAFE algorithm has different performance under different antenna number, as shown in Figure 7. From Figure 7, we find that angle and 
frequency estimation performance of MDJAFE algorithm is improved with $M$ increasing.

Simulation 6. The performance of this algorithm under different delay number $P$ is investigated in the simulation. $M=12, K=3$, and $N=400$ in this simulation. The delay number $P$ is set to $1,2,3$, and 4 . MDJAFE algorithm has different performance under different delay numbers, as shown in Figure 8. From Figure 8, we find that angle and frequency estimation performance of MDJAFE algorithm is improved with $P$ increasing.

\section{Conclusion}

This work presents a new ESPRIT algorithm-based joint angle and frequency estimation using multiple-delay output. The advantage of this proposed algorithm using the multiple-delay output over the conventional algorithm is that the estimation accuracy has been greatly improved.

\section{Acknowledgments}

This paper is supported by China NSF Grant (60801052), Aeronautical Science Foundation of China (2009ZC52036), Ph.D. Programs Foundation of China's Ministry of Education (200802871056) and Nanjing University of Aeronautics and Astronautics Research Funding (NS2010109, NS2010114). The authors thank Professor Zhang Xiaofei for his help.

\section{References}

[1] B. Widrow and S. D. Stearns, Adaptive Signal Processing, Prentice-Hall, Englewood Cliffs, NJ, USA, 1985.

[2] X. Zhang and D. Xu, "Improved coherent DOA estimation algorithm for uniform linear arrays," International Journal of Electronics, vol. 96, no. 2, pp. 213-222, 2009.

[3] X. Zhang, X. Gao, and D. Xu, "Multi-invariance ESPRIT-based blind DOA estimation for MC-CDMA with an antenna array," IEEE Transactions on Vehicular Technology, vol. 58, no. 8, pp. 4686-4690, 2009.

[4] X. Zhang, X. Gao, and W. Chen, "Improved blind 2Ddirection of arrival estimation with L-shaped array using shift invariance property," Journal of Electromagnetic Waves and Applications, vol. 23, no. 5, pp. 593-606, 2009.

[5] X. Zhang, J. Yu, G. Feng, and D. Xu, "Blind direction of arrival estimation of coherent sources using multi-invariance property," Progress In Electromagnetics Research, vol. 88, pp. 181-195, 2008.

[6] E. Rosnes and A. Vahlin, "Frequency estimation of a single complex sinusoid using a generalized Kay estimator," IEEE Transactions on Communications, vol. 54, no. 3, pp. 407-415, 2006.

[7] X. F. Zhang and D. Z. Xu, "Novel joint time delay and frequency estimation method," IET Radar, Sonar and Navigation, vol. 3, no. 2, pp. 186-194, 2009.

[8] J. Tsui, Digital Techniques for Wideband Receivers, chapter 12, Artech House, Norwood, Mass, USA, 2nd edition, 2001.

[9] M. Djeddou, A. Belouchrani, and S. Aouada, "Maximum likelihood angle-frequency estimation in partially known correlated noise for low-elevation targets," IEEE Transactions on Signal Processing, vol. 53, no. 8, pp. 3057-3064, 2005.

[10] M. D. Zoltowski and C. P. Mathews, "Real-time frequency and 2-D angle estimation with sub-Nyquistspatio-temporal sampling," IEEE Transactions on Signal Processing, vol. 42, no. 10, pp. 2781-2794, 1994.

[11] M. Haardt and J. A. Nossek, "3-D unitary ESPRIT for joint 2-D angle and carrier estimation," in Proceedings of the IEEE International Conference on Acoustics, Speech, and Signal Processing (ICASSP '97), pp. 255-258, Munich, Germany, April 1997.

[12] A. N. Lemma, A. J. van der Veen, and E. F. Deprettere, "Joint angle-frequency estimation using multi-resolution ESPRIT," in Proceedings of the IEEE International Conference on Acoustics, Speech and Signal Processing (ICASSP '98), vol. 4, pp. 1957-1960, Seattle, Wash, USA, May 1998.

[13] A. N. Lemma, A. J. van der Veen, and ED. F. Deprettere, "Analysis of joint angle-frequency estimation using ESPRIT," IEEE Transactions on Signal Processing, vol. 51, no. 5, pp. 12641283, 2003.

[14] S. Wang and X. Zhou, "Direction-of-arrival and frequency estimation in array signal processing," Journal of Shanghai Jiaotong University, vol. 33, no. 1, pp. 40-42, 1999.

[15] Z. Xiaofei, F. Gaopeng, Y. Jun, and X. Dazhuan, "Anglefrequency estimation using trilinear decomposition of the oversampled output," Wireless Personal Communications, vol. 51, no. 2, pp. 365-373, 2009.

[16] X. Zhang, D. Wang, and D. Xu, "Novel blind joint direction of arrival and frequency estimation for uniform linear array," Progress in Electromagnetics Research, vol. 86, pp. 199-215, 2008.

[17] H. Chen, Y. Wang, and Z. Wu, "Frequency and 2-D angle estimation based on uniform circular array," in Proceedings of IEEE International Symposium on Phased Array Systems and Technology, pp. 547-552, 2003.

[18] T. Fu, S. Jin, and X. Gao, "Joint 2-D angle and frequency estimation for uniform circular array," in Proceedings of International Conference on Communications, Circuits and Systems (ICCCAS '06), vol. 1, pp. 230-233, June 2006.

[19] W. Jia, M. Yao, and J. Song, "Joint frequency, two dimensional arrival angles estimations via marked signal subspace," in Proceedings of the 8th International Conference on Signal Processing (ICSP '06), vol. 1, pp. 16-20, November 2006.

[20] C. H. Lin, W. H. Fang, K. H. Wu, and J. D. Lin, "Fast algorithm for joint azimuth and elevation angles, and frequency estimation via hierarchical space-time decomposition," in Proceedings of IEEE International Conference on Acoustics, Speech and Signal Processing (ICASSP '07), vol. 2, pp. 10611064, April 2007.

[21] M. A. Hasan, "DOA and frequency estimation using fast subspace algorithms," Signal Processing, vol. 77, no. 1, pp. 4962, 1999.

[22] H. Jiacai, S. Yaowu, and T. Jianwu, "Joint estimation of DOA, frequency, and polarization based on cumulants and UCA," Journal of Systems Engineering and Electronics, vol. 18, no. 4, pp. 704-709, 2007.

[23] J. Liang, X. Zeng, B. Ji, J. Zhang, and F. Zhao, "A computationally efficient algorithm for joint range-DOA-frequency estimation of near-field sources," Digital Signal Processing, vol. 19, no. 4, pp. 596-611, 2009.

[24] M. Amin and Y. Zhang, "Spatial time-frequency distributions and DOA estimation," in Classical and Modern Direction-ofArrival Estimation, pp. 185-217, 2009. 